\title{
TUTTE POLYNOMIALS AND BICYCLE DIMENSION OF TERNARY MATROIDS
}

\author{
FRANCOIS JAEGER
}

(Communicated by Thomas Brylawski)

\begin{abstract}
Let $M$ be a ternary matroid, $t(M, x, y)$ be its Tutte polynomial and $d(M)$ be the dimension of the bicycle space of any representation of $M$ over $\mathrm{GF}(3)$.

We show that, for $j=e^{2 i \pi / 3}$, the modulus of the complex number $t\left(M, j, j^{2}\right)$ is equal to $(\sqrt{ } 3)^{d(M)}$. The proof relies on the study of the weight enumerator $W_{f}(y)$ of the cycle space $\mathscr{E}$ of a representation of $M$ over $\mathrm{GF}(3)$ evaluated at $y=j$. The main tool is the concept of principal quadripartition of $\mathscr{h}$ which allows a precise analysis of the evolution of the relevant invariants under deletion and contraction of elements.
\end{abstract}

Resume. Soit $M$ un matroïde ternaire, $t(M, x, y)$ son polynôme de Tutte et $d(M)$ la dimension de l'espace des bicycles d'une représentation quelconque de $M$ sur $\mathrm{GF}(3)$.

Nous montrons que, pour $j=e^{2 i \pi / 3}$, le module du nombre complexe $t\left(M, j, j^{2}\right)$ est égal à $(\sqrt{ } 3)^{d(M)}$. La preuve s'appuie sur l'étude de l'énumérateur de poids $W_{i \in}(y)$ de l'espace des cycles $\mathscr{C}$ d'une représentation de $M$ sur $\mathrm{GF}(3)$ pour la valeur $y=j$. L'outil essentiel est le concept de quadripartition principale de $\mathscr{C}$ qui permet une analyse précise de l'évolution des invariants concernés relativement à la suppression ou contraction d'éléments.

\section{INTRODUCTION}

The initial motivation for the present work was a discussion with Tom Brylawski (London, July 1987). He pointed out that, since all representations of a matroid $M$ over $F$ (where $F$ is $\mathrm{GF}(2)$ or $\operatorname{GF}(3))$ are essentially equivalent (see [2]), the dimension of the bicycle space (the intersection of the cycle and cocycle spaces) of any such representation depends only on $M$. Let us denote (when $M$ is representable over $F$ ) this dimension by $d(M, F)$. When $F$ is $\operatorname{GF}(2), d(M, F)$ can be obtained from the Tutte polynomial $t(M, x, y)$ of $M$. More precisely, if $M$ is a binary matroid on the set $E$, $t(M,-1,-1)=(-1)^{|E|}(-2)^{d(M, \mathrm{GF}(2))}$ (this is proved in [8, Theorem 9.1]

Received by the editors August 28, 1988.

1980 Mathematics Subject Classification (1985 Revision). Primary 05B35; Secondary 05A 15.

Work partially supported by PRC Mathématiques et Informatique. 
for graphic matroids, and the extension of the proof to arbitrary binary matroids is immediate). Brylawski asked whether a similar formula existed for $d(M, \mathrm{GF}(3))$. The present paper gives a positive answer to this question.

The essential clue in this research was the realization that a knot-theoretic result of Lickorish and Millett [6, Theorem 3], restricted to alternating links, gives the solution of Brylawski's question for cycle matroids of plane graphs. This result relates the Jones polynomial $V_{L}(t)$ of a link $L$ evaluated at $t=e^{i \pi / 3}$ to the dimension of the $\mathrm{GF}(3)$-homology $H_{1}\left(D_{L}, \mathrm{GF}(3)\right)$ of the double cyclic cover of $S^{3}$ branched along $L$. For alternating links, the Jones polynomial is closely related to the Tutte polynomial of an associated plane graph $G$ (see for instance [5], [9]), and (using an appropriate presentation matrix) one can also interpret $H_{1}\left(D_{L}, \mathrm{GF}(3)\right)$ as the bicycle space of $G$ over $\mathrm{GF}(3)$. In fact it can be checked that the restriction of our main result to cycle matroids of plane graphs is equivalent to the restriction of the Lickorish-Millett result to alternating links. A detailed account of this connection between the two results, whose proofs are entirely different, is beyong the scope of the present paper.

The paper is organized as follows. In Section 2 we work in the context of spaces over $\mathrm{GF}(3)$ and no matroid-theoretic knowledge is required. We introduce the principal quadripartition of a ternary space and use it to study the modification of the bicycle dimension under deletion and contraction of elements. Then we relate the bicycle dimension of a ternary space to a special value of its weight enumerator. Section 3 reformulates the previous results in matroid-theoretic terms, using Greene's formula [4] which relates the weight enumerator of a space to the Tutte polynomial of the associated matroid. Finally some concluding remarks are presented in Section 4.

\section{BICYCLE DIMENSION AND WEIGHT ENUMERATION IN TERNARY SPACES}

2.1 Definitions, notations. Let $E$ be a finite nonempty set. The set $\operatorname{GF}(3)^{E}$ of mappings from $E$ to $\mathrm{GF}(3)$ is endowed with its canonical structure of vector space over $\mathrm{GF}(3)$. For every mapping $C$ in $\mathrm{GF}(3)^{E}$ the support of $C$, denoted by $S(C)$, is the set of elements $e$ of $E$ such that $C(e) \neq 0$. We shall denote $|S(C)|$ by $s(C)$. For every element $e$ of $E$ we denote by $\underline{e}$ the mapping from $E$ to $\mathrm{GF}(3)$ which takes the value 1 on $e$ and the value 0 on $E-\{e\}$. For $C$ and $K$ in $\operatorname{GF}(3)^{E}$ we write $\langle C, K\rangle=\sum_{e \in E} C(e) K(e)$, and $C, K$ are said to be orthogonal if $\langle C, K\rangle=0$.

We call ternary space on $E$, or more simply in this section space on $E$, any subspace of $\operatorname{GF}(3)^{E}$. For any space $\mathscr{C}$ on $E$, we denote by $\mathscr{C}^{\perp}$ the space on $E$ formed by the elements of $\mathrm{GF}(3)^{E}$ orthogonal to all elements of $\mathscr{C}$, by $\mathscr{B}(\mathscr{C})$ the space $\mathscr{C} \cap \mathscr{C}^{\perp}$ on $E$ and by $d(\mathscr{C})$ the dimension of $\mathscr{B}(\mathscr{C})$, which we call the bicycle dimension of $\mathscr{C}$. A loop of $\mathscr{C}$ is an element $e$ of $E$ such that $\underline{e} \in \mathscr{C}$, and a coloop of $\mathscr{C}$ is a loop of $\mathscr{C}^{\perp}$. For $|E| \geq 2$ and $e$ in $E$, we denote by $\mathscr{C}-e$ the space $\{C \cdot e / C \in \mathscr{C}, C(e)=0\}$ on $E-\{e\}$ and by $\mathscr{C} \cdot e$ the space $\{C \cdot e / C \in \mathscr{C}\}$ on $E-\{e\}$, where $C \cdot e$ denotes the 
restriction of $C$ to $E-\{e\}$. Then it is easy to check that $(\mathscr{C}-e)^{\perp}=\mathscr{C}^{\perp} \cdot e$ and $(\mathscr{C} \cdot e)^{\perp}=\mathscr{C}^{\perp}-e$. In the sequel whenever the notations $\mathscr{C}-e$ and $\mathscr{C} \cdot e$ are used it is implicitly assumed that $|E| \geq 2$.

2.2 The principal quadripartition and bicycle dimension. Let $E$ be a finite nonempty set and $\mathscr{C}$ be a space on $E$. The following result is analogous to the tripartition theorem given in [8, Theorem 2.1] for binary cycle spaces of graphs (and it can obviously be extended to spaces over any finite field).

Proposition 1. For every element $e$ of $E$ exactly one of the following properties holds:

(1) there exists $B$ in $\mathscr{B}(\mathscr{C})$ with $B(e) \neq 0$;

(2a) there exists $C$ in $\mathscr{C}$ and $K$ in $\mathscr{C}^{\perp}$ with $\underline{e}=C+K$ and $C(e)=1$, $K(e)=0$

(2b) there exists $C$ in $\mathscr{C}$ and $K$ in $\mathscr{C}^{\perp}$ with $\underline{e}=C+K$ and $C(e)=0$, $K(e)=1$;

(2c) there exists $C$ in $\mathscr{C}$ and $K$ in $\mathscr{C}^{\perp}$ with $\underline{e}=C+K$ and $C(e)=-1$, $K(e)=-1$

Proof. Consider the property: (2) $\underline{e} \in \mathscr{C}+\mathscr{C}^{\perp}$. Since $\left(\mathscr{C}+\mathscr{C}^{\perp}\right)^{\perp}=$ $\mathscr{C}^{\perp} \cap\left(\mathscr{C}^{\perp}\right)^{\perp}=\mathscr{C}^{\perp} \cap \mathscr{C}$, (2) holds if and only if $\underline{e}$ is orthogonal to every $B$ in $\mathscr{B}(\mathscr{C})$. Hence (2) is the negation of (1). On the other hand, (2) holds if and only if $(2 a),(2 b)$ or $(2 c)$ holds. Thus it remains to show that $(2 a),(2 b)$ and (2c) are mutually exclusive. Assume that (2) holds and that $\underline{e}=C+K=C^{\prime}+K^{\prime}$ with $C, C^{\prime}$ in $\mathscr{C}$ and $K, K^{\prime}$ in $\mathscr{C}^{\perp}$. Then $C-C^{\prime}=K^{\prime}-K$ is an element $B$ of $\mathscr{B}(\mathscr{C})$. Since $(1)$ does not hold, $B(e)=0$ and hence $C(e)=C^{\prime}(e)$ and $K(e)=K^{\prime}(e)$. This completes the proof.

We shall say that the element $e$ of $E$ is of type (1), (2a), (2b), or (2c) (with respect to $\mathscr{C}$ ) if $e$ satisfies the corresponding property in Proposition 1 .

Remark. A loop is of type (2a) and a coloop is of type (2b).

Proposition 2. If $e$ is a loop or coloop, $d(\mathscr{C}-e)=d(\mathscr{C} \cdot e)=d(\mathscr{C})$. Otherwise:

(1) If $e$ is of type $(1)$, then $d(\mathscr{C}-e)=d(\mathscr{C})-1$ and $d(\mathscr{C} \cdot e)=d(\mathscr{C})-1$.

(2a) If $e$ is of type $(2 a)$, then $d(\mathscr{C}-e)=d(\mathscr{C})$ and $d(\mathscr{C} \cdot e)=d(\mathscr{C})+1$.

(2b) If $e$ is of type $(2 b)$, then $d(\mathscr{C}-e)=d(\mathscr{C})+1$ and $d(\mathscr{C} \cdot e)=d(\mathscr{C})$.

(2c) If $e$ is of type $(2 c)$, then $d(\mathscr{C}-e)=d(\mathscr{C})$ and $d(\mathscr{C} \cdot e)=d(\mathscr{C})$.

Proof. First note that $\mathscr{B}(\mathscr{C}-e)=(\mathscr{C}-e) \cap(\mathscr{C}-e)^{\perp}=(\mathscr{C}-e) \cap \mathscr{C}^{\perp} \cdot e=$ $\left\{K \cdot e / K \in \mathscr{C}^{\perp},(K \cdot e)^{\wedge} \in \mathscr{C}\right\}$, where $(K \cdot e)^{\wedge}$ is the mapping from $E$ to $\operatorname{GF}(3)$ which coincides with $K \cdot e$ on $E-\{e\}$ and takes the value 0 on $e$. Clearly $\mathscr{B}(\mathscr{C})-e=\left\{B \cdot e / B \in \mathscr{C}^{\perp}, B \in \mathscr{C}, B(e)=0\right\}$ is a subspace of $\mathscr{B}(\mathscr{C}-e)$. An element of $\mathscr{B}(\mathscr{C}-e)-(\mathscr{B}(\mathscr{C})-e)$ is of the form $K \cdot e$ for some $K \in \mathscr{C}^{\perp}$ with $(K \cdot e)^{\wedge} \in \mathscr{C}$ and $K(e) \neq 0$. Since $\underline{e}=K(e) K-K(e)(K \cdot e)^{\wedge}$, $e$ must then be of type $(2$ b) with respect to $\mathscr{C}$. 
Thus if $e$ is not of type $(2 \mathrm{~b}), \mathscr{B}(\mathscr{C}-e)=\mathscr{B}(\mathscr{C})-e$. Hence $d(\mathscr{C}-e)=$ $\operatorname{dim} \mathscr{B}(\mathscr{C}) \cap \mathscr{H}$, where $\mathscr{H}$ is the hyperplane orthogonal to $\underline{e}$. It follows that $d(\mathscr{C}-e)=d(\mathscr{C})-1$ if $e$ is of type (1), and $d(\mathscr{C}-e)=d(\mathscr{C})$ if $e$ is of type (2a) or $(2 \mathrm{c})$.

If $e$ is of type (2b) there exists $C_{0}$ in $\mathscr{C}$ and $K_{0}$ in $\mathscr{C}^{\perp}$ with $\underline{e}=C_{0}+K_{0}$ and $C_{0}(e)=0, K_{0}(e)=1$. Then $\left(K_{0} \cdot e\right)^{\wedge}=-C_{0}$ belongs to $\mathscr{C}$ and hence $K_{0} \cdot e$ belongs to $\mathscr{B}(\mathscr{C}-e)$. Moreover if $K \cdot e$ belongs to $\mathscr{B}(\mathscr{C}-e)-(\mathscr{B}(\mathscr{C})-e)$ (so that $K \in \mathscr{C}^{\perp},(K \cdot e)^{\wedge} \in \mathscr{C}$ and $\left.K(e) \neq 0\right), K(e) K-K(e)(K \cdot e)^{\wedge}=\underline{e}=$ $C_{0}+K_{0}$. Hence $B=K(e) K-K_{0}=C_{0}+K(e)(K \cdot e)^{\wedge}$ belongs to $\mathscr{B}(\mathscr{C})$ and takes the value zero on $e$. Then $K(e) K \cdot e-K_{0} \cdot e=B \cdot e$ belongs to $\mathscr{B}(\mathscr{C})-e$. It follows that $\mathscr{B}(\mathscr{C}-e)=(\mathscr{B}(\mathscr{C})-e)+\mathscr{S}$, where $\mathscr{S}$ is the space generated by $K_{0} \cdot e$. Note that $K_{0} \cdot e$ belongs to $(\mathscr{B}(\mathscr{C})-e)$ if and only if $K_{0} \cdot e=B \cdot e$ for some $B \in \mathscr{B}(\mathscr{C})$ with $B(e)=0$. In this case $\underline{e}=K_{0}-B$ belongs to $\mathscr{C}^{\perp}$ and hence $e$ is a coloop of $\mathscr{C}$. Conversely if $e$ is a coloop of $\mathscr{C}, C_{0}=\underline{e}-K_{0}$ belongs to $\mathscr{B}(\mathscr{C}), C_{0}(e)=0$ and $K_{0} \cdot e=-C_{0} \cdot e$, so that $K_{0} \cdot e$ belongs to $(\mathscr{B}(\mathscr{C})-e)$. It follows that $d(\mathscr{C}-e)=\operatorname{dim}(\mathscr{B}(\mathscr{C})-e)$ if $e$ is a coloop, and $d(\mathscr{C}-e)=\operatorname{dim}(\mathscr{B}(\mathscr{C})-e)+1$ otherwise. Since $e$ is not of type (1), $\operatorname{dim}(\mathscr{B}(\mathscr{C})-e)=\operatorname{dim} \mathscr{B}(\mathscr{C}) \cap \mathscr{H}=\operatorname{dim} \mathscr{B}(\mathscr{C})$. This completes the proof of the evaluation of $d(\mathscr{C}-e)$.

For the evaluation of $d(\mathscr{C} \cdot e)$, we observe that $d(\mathscr{C})=d\left(\mathscr{C}^{\perp}\right), d(\mathscr{C} \cdot e)=$ $d\left((\mathscr{C} \cdot e)^{\perp}\right)=d\left(\mathscr{C}^{\perp}-e\right)$ and we apply the above results to $\mathscr{C}^{\perp}$. We also use the obvious facts that $e$ is of type (1) (respectively: (2a), (2b) (2c)) with respect to $\mathscr{C}$ if and only if it is of type (1) (respectively: (2b), (2a), (2c)) with respect to $\mathscr{C}^{\perp}$, and that $e$ is a loop (respectively: coloop) of $\mathscr{C}$ if and only if it is a coloop (respectively: loop) of $\mathscr{C}^{\perp}$.

2.3 The main result. Let $j=e^{2 i \pi / 3}$, so that $j^{3}=1$ and $1+j+j^{2}=0$. To every space $\mathscr{C}$ on the finite nonempty set $E$ we associate the complex number $f(\mathscr{C})=\sum_{C \in \mathscr{F}} j^{s(C)}$, which is the evaluation at $y=j$ of the weight enumerator $W_{f}(y)$ of $\mathscr{C}$ considered as a linear code (see [7, Chapter 5]).

Proposition 3. Let $\mathscr{C}$ be a space on $E$ and e be an element of $E$ of type (2b) with respect to $\mathscr{C}$. Then $f(\mathscr{C})=f(\mathscr{C} \cdot e)$.

Proof. Clearly

$$
f(\mathscr{C})=\sum_{C \in \mathscr{F}, C(e)=0} j^{s(C)}+2 \sum_{C \in \mathscr{F}, C(e)=1} j^{s(C)} .
$$

Since $e$ is not a loop, every element of $\mathscr{C} \cdot e$ is of the form $C \cdot e$ for a unique element $C$ of $\mathscr{C}$ (because if $C \cdot e=C^{\prime} \cdot e, S\left(C-C^{\prime}\right) \subseteq\{e\}$ implies $C=C^{\prime}$ ). It follows that:

$$
f(\mathscr{C} \cdot e)=\sum_{C \in \mathscr{F}} j^{s(C \cdot e)}=\sum_{C \in \mathscr{F}, C(e)=0} j^{s(C)}+2 \sum_{C \in \mathscr{F}, C(e)=1} j^{s(C)-1} .
$$

We now prove that $g(\mathscr{C})=\sum_{C \in \mathscr{F}, C(e)=1} j^{s(C)}=0$. Let $Z$ be an element of $\mathscr{C}$ with $Z(e)=0$ such that $\underline{e}=Z+K$ for some $K$ in $\mathscr{C}^{\perp}$. Then, since 
$\{C \in \mathscr{C}, C(e)=1\}$ is invariant under a translation of vector $Z$ :

$$
3 g(\mathscr{C})=\sum_{C \in \mathscr{F}, C(e)=1} j^{s(C)}+j^{s(C+Z)}+j^{s(C-Z)} \text {. }
$$

For every $C \in \mathscr{C}$ we write $C_{0}=\{x \in E / C(x) \neq 0, Z(x)=0\}, C_{+}=$ $\{x \in E / C(x)=Z(x) \neq 0\}, C_{-}=\{x \in E / C(x)=-Z(x) \neq 0\}, Z_{0}=$ $\{x \in E / C(x)=0, Z(x) \neq 0\}$. Then $s(C)=\left|C_{0}\right|+\left|C_{+}\right|+\left|C_{-}\right|, S(C+Z)=$ $\left|C_{0}\right|+\left|C_{+}\right|+\left|Z_{0}\right|$ and $s(C-Z)=\left|C_{0}\right|+\left|C_{-}\right|+\left|Z_{0}\right|$.

Let $C$ be an element of $\mathscr{C}$ with $C(e)=1$. Since $0=\langle C, K\rangle=\langle C, \underline{e}-Z\rangle=$ $1-\langle C, Z\rangle$ we have $\left|C_{+}\right|-\left|C_{-}\right| \equiv 1(\bmod 3)$. Similarly, since $0=\langle Z, K\rangle=$ $\langle Z, \underline{e}-Z\rangle=-\langle Z, Z\rangle$ we have $s(Z)=\left|Z_{0}\right|+\left|C_{+}\right|+\left|C_{-}\right| \equiv 0 \quad(\bmod 3)$.

Now $j^{s(C)}+j^{s(C+Z)}+j^{s(C-Z)}=j^{\left|C_{0}\right|}\left(j^{\left|C_{+}\right|+\left|C_{-}\right|}+j^{\left|C_{+}\right|+\left|Z_{0}\right|}+j^{\left|C_{-}\right|+\left|Z_{0}\right|}\right)$. Since $\left|C_{+}\right|+\left|C_{-}\right| \equiv 2\left|C_{-}\right|+1 \quad(\bmod 3),\left|C_{+}\right|+\left|Z_{0}\right| \equiv 2\left|C_{-}\right|(\bmod 3),\left|C_{-}\right|+\left|Z_{0}\right| \equiv$ $2\left|C_{-}\right|-1(\bmod 3)$ and $1+j+j^{2}=0, j^{s(C)}+j^{s(C+Z)}+j^{s(C-Z)}=0$. It follows that $g(\mathscr{C})=0$ as required.

Proposition 4. Let $\mathscr{C}$ be a space on $E$ and $e$ be an element of $E$ which is not a loop of $\mathscr{C}$. Then

(1) If $e$ is of type (1) with respect to $\mathscr{C}, f(\mathscr{C})=\left(j-j^{2}\right) f(\mathscr{C} \cdot e)$.

(2a) If $e$ is of type $(2 a)$ with respect to $\mathscr{C}, f(\mathscr{C})=\left(\left(j-j^{2}\right) / 3\right) f(\mathscr{C} \cdot e)$.

(2c) If $e$ is of type (2c) with respect to $\mathscr{C}, f(\mathscr{C})=-f(\mathscr{C} \cdot e)$.

Proof. Let $Z$ be an element of $\mathscr{C}$ with $Z(e) \neq 0$ (which exists since $e$ is not of type $(2 b)$ and hence not a coloop), $\mathscr{Z}$ be the space generated by $Z$, and $\mathscr{H}$ be the hyperplane orthogonal to $\underline{e}$. Then clearly $\mathscr{C}=(\mathscr{C} \cap \mathscr{H}) \oplus \mathscr{Z}$, where $\oplus$ denotes the direct sum of vector spaces. It follows that:

$$
f(\mathscr{C})=\sum_{C \in \mathscr{F} \cap \mathscr{P}} j^{s(C)}+j^{s(C+Z)}+j^{s(C-Z)} .
$$

Now, since every element of $\mathscr{C} \cdot e$ is of the form $C \cdot e$ for a unique element $C$ of $\mathscr{C}$, we may write:

$$
\begin{aligned}
f(\mathscr{C} \cdot e) & =\sum_{C \in \mathscr{C} \cap \mathscr{P}} j^{s(C \cdot e)}+j^{s((C+Z) \cdot e)}+j^{s((C-Z) \cdot e)} \\
& =\sum_{C \in \mathscr{E} \cap \mathscr{H}} j^{s(C)}+j^{s(C+Z)-1}+j^{s(C-Z)-1} .
\end{aligned}
$$

For every $C$ in $\mathscr{C}$ we define $C_{0}, C_{+}, C_{-}, Z_{0}$ as in the proof of Proposition 3, so that $s(C)=\left|C_{0}\right|+\left|C_{+}\right|+\left|C_{-}\right|, s(C+Z)=\left|C_{0}\right|+\left|C_{+}\right|+\left|Z_{0}\right|$ and $s(C-Z)=\left|C_{0}\right|+\left|C_{-}\right|+\left|Z_{0}\right|$. It follows that

(i) $f(\mathscr{C})=\sum_{C \in \mathscr{E} \cap \not{H}} j^{s(C)}\left(1+j^{\left|Z_{0}\right|-\left|C_{-}\right|}+j^{\left|Z_{0}\right|-\left|C_{+}\right|}\right)$;

(ii) $f(\mathscr{C} \cdot e)=\sum_{C \in \mathscr{F} \cap \mathscr{P}} j^{s(C)}\left(1+j^{\left|Z_{0}\right|-\left|C_{-}\right|-1}+j^{\left|Z_{0}\right|-\left|C_{+}\right|-1}\right)$.

(1). If $e$ is of type (1) with respect to $\mathscr{C}$, we take for $Z$ an element of $\mathscr{C} \cap \mathscr{C}^{\perp}$ with $Z(e) \neq 0$. Then for any $C$ in $\mathscr{C} \cap \mathscr{H}, Z$ is orthogonal to $C$ and hence 
$\left|C_{+}\right| \equiv\left|C_{-}\right| \quad(\bmod 3)$. Moreover, since $Z$ is orthogonal to itself, $\left|Z_{0}\right|+\left|C_{+}\right|+$ $\left|C_{-}\right| \equiv 0(\bmod 3)$ and hence $\left|Z_{0}\right| \equiv\left|C_{+}\right| \equiv\left|C_{-}\right|(\bmod 3)$. It follows that $1+j^{\left|Z_{0}\right|-\left|C_{-}\right|}+j^{\left|Z_{0}\right|-\left|C_{1}\right|}=3$ and $1+j^{\left|Z_{0}\right|-\left|C_{-}\right|-1}+j^{\left|Z_{0}\right|-\left|C_{+}\right|-1}=1+2 j^{2}$. Since $3=\left(j-j^{2}\right)\left(1+2 j^{2}\right)$, it follows from (i) and (ii) that $f(\mathscr{C})=\left(j-j^{2}\right) f(\mathscr{C} \cdot e)$. $(2 a)$. If $e$ is of type $(2 a)$ with respect to $\mathscr{C}$, we take for $Z$ an element of $\mathscr{C}$ with $Z(e)=1$ and $\underline{e}=Z+K$ for some $K$ in $\mathscr{C}^{\perp}$. Then for any $C$ in $\mathscr{C} \cap \mathscr{H}, 0=\langle C, K\rangle=\langle C, \underline{e}-Z\rangle=-\langle C, Z\rangle$ and hence $\left|C_{+}\right| \equiv\left|C_{-}\right|(\bmod 3)$. Similarly, since $0=\langle Z, K\rangle=\langle Z, \underline{e}-Z\rangle=1-\langle Z, Z\rangle,\left|Z_{0}\right|+\left|C_{+}\right|+\left|C_{-}\right| \equiv$ $1(\bmod 3)$ and hence $\left|Z_{0}\right|-\left|C_{+}\right| \equiv\left|Z_{0}\right|-\left|C_{-}\right| \equiv 1 \quad(\bmod 3)$. It follows that $1+j^{\left|Z_{1}\right|-\left|C_{-}\right|}+j^{\left|Z_{1}\right|-\left|C^{\prime}\right|}=1+2 j=j-j^{2}$ and $1+j^{\left|Z_{0}\right|-\left|C_{-}\right|-1}+j^{\left|Z_{0}\right|-\left|C_{+}\right|-1}=3$. Hence, by (i) and (ii), $f(\mathscr{C})=\left(\left(j-j^{2}\right) / 3\right) f(\mathscr{C} \cdot e)$.

$(2 c)$. If $e$ is of type $(2 c)$ with respect to $\mathscr{C}$, we take for $Z$ an element of $\mathscr{C}$ with $Z(e)=-1$ and $\underline{e}=Z+K$ for some $K$ in $\mathscr{C}^{\perp}$. As before, for any $C$ in $\mathscr{C} \cap \mathscr{H},\left|C_{+}\right| \equiv\left|C_{-}\right|(\bmod 3)$. Moreover $0=\langle Z, \underline{e}-Z\rangle=$ $-1-\langle Z, Z\rangle$ implies $\left|Z_{0}\right|+\left|C_{+}\right|+\left|C_{-}\right| \equiv 2(\bmod 3)$ and hence $\left|Z_{0}\right|-\left|C_{+}\right| \equiv$ $\left|Z_{0}\right|-\left|C_{-}\right| \equiv 2(\bmod 3)$. It follows that $1+j^{\left|Z_{0}\right|-\left|C_{-}\right|}+j^{\left|Z_{0}\right|-\left|C_{+}\right|}=1+2 j^{2}$ and $1+j^{\left|Z_{0}\right|-\left|C_{-}\right|-1}+j^{\left|Z_{0}\right|-|C \cdot|-1}=1+2 j=-1-2 j^{2}$. Hence, by (i) and (ii), $f(\mathscr{C})=-f(\mathscr{C} \cdot e)$.

For every space $\mathscr{C}$ on $E$, let $\varepsilon(\mathscr{C})=\left(j-j^{2}\right)^{-\operatorname{dim} \mathscr{F}-d(\mathscr{F})} f(\mathscr{C})$.

Proposition 5. For every space $\mathscr{C}$ on $E, \varepsilon(\mathscr{C})=\varepsilon(\mathscr{C} \cdot e)$ if $e$ is a loop or is of type (1) or $(2 b)$ with respect to $\mathscr{C}$, and $\varepsilon(\mathscr{C})=-\varepsilon(\mathscr{C} \cdot e)$ otherwise.

Proof. If $e$ is a loop, $\mathscr{C}=(\mathscr{C} \cap \mathscr{H}) \oplus \mathscr{Z}$, where $\mathscr{Z}$ is the space generated by $\underline{e}$ and $\mathscr{H}$ is the hyperplane orthogonal to $\underline{e}$. Hence $f(\mathscr{C})=$ $\sum_{C \in \mathscr{C} \cap \mathscr{F}} j^{s(C)}+j^{s(C+\underline{e})}+j^{s(C-\underline{e})}$ and $f(\mathscr{C} \cdot e)=\sum_{C \in \mathscr{F} \cap \mathscr{P}} j^{s(C)}$. It follows that $f(\mathscr{C})=(1+2 j) f(\mathscr{C} \cdot e)=\left(j-j^{2}\right) f(\mathscr{C} \cdot e)$. Clearly $\operatorname{dim} \mathscr{C}=\operatorname{dim}(\mathscr{C} \cdot e)+1$ and, by Proposition $2, d(\mathscr{C})=d(\mathscr{C} \cdot e)$. Hence $\varepsilon(\mathscr{C})=\varepsilon(\mathscr{C} \cdot e)$.

If $e$ is not a loop, $\operatorname{dim} \mathscr{C}=\operatorname{dim}(\mathscr{C} \cdot e)$ and the result follows easily from Propositions 2, 3, 4.

Proposition 6. For every space $\mathscr{C}$ on $E, \quad \sum_{C \in \mathscr{F}} j^{s(C)}=f(\mathscr{C})=$ $\varepsilon(\mathscr{C})\left(j-j^{2}\right)^{\operatorname{dim} t+d(\mathscr{t})}$ with $\varepsilon(\mathscr{C}) \in\{1,-1\}$. Equivalently, $(f(\mathscr{C}))^{2}=$ $(-3)^{\operatorname{dim} t+d(t)}$.

Proof. If $|E|=1$ and $e$ is a loop of $\mathscr{C}, \operatorname{dim} \mathscr{C}=1, d(\mathscr{C})=0$ and $f(\mathscr{C})=$ $1+2 j=j-j^{2}$, so that $\varepsilon(\mathscr{C})=1$. If $|E|=1$ and $e$ is a coloop of $\mathscr{C}$, $\operatorname{dim} \mathscr{C}=0, d(\mathscr{C})=0$ and $f(\mathscr{C})=1$, so that $\varepsilon(\mathscr{C})=1$ also in this case. Then the result follows by induction using Proposition 5.

\section{BiCyCle Dimension AND TUtTE POLyNOMials}

Now we need some basic notions of matroid theory which can be found in [12]. Let $E$ be a finite nonempty set, $\mathscr{C}$ be a subspace of $\operatorname{GF}(q)^{E}$ and $S(\mathscr{C})$ 
be the set of supports of elements of $\mathscr{C}$ (the definitions and notations are the same as those at the beginning of Section 2). The elements of $S(\mathscr{C})-\{\varnothing\}$ which are minimal by inclusion form the circuits of a matroid on $E$ which we denote by $M(\mathscr{C})$. Then $\mathscr{C}$ is the cycle space of an appropriate representation $R$ of $M(\mathscr{C})$ over $\operatorname{GF}(q), \mathscr{C}^{\perp}$ is the cocycle space of $R$ and $M(\mathscr{C})$ and $M\left(\mathscr{C}^{\perp}\right)$ are dual matroids. The vector space $\mathscr{C} \cap \mathscr{C}^{\perp}$ is usually called the bicycle space of the representation $R$. A matroid is said to be ternary if it is of the form $M(\mathscr{C})$ for some ternary space $\mathscr{C}$.

Let $M$ be a matroid on $E$ with rank-function $r$. The Tutte polynomial of $M$ (first introduced for graphic matroids in [10], [11] and then for matroids in [1] and [3]) is the polynomial $t(M, x, y)$ in two variables $x, y$ defined by

$$
t(M, x, y)=\sum_{F \subseteq E}(x-1)^{r(E)-r(F)}(y-1)^{|F|-r(F)} .
$$

This polynomial satisfies the following properties which yield a recursive definition:

(i) If $|E|=1$ : if the unique element of $E$ is a coloop of $M, t(M, x, y)=$ $x$; if it is a loop of $M, t(M, x, y)=y$.

(ii) If the element $e$ of $E$ is neither a loop nor a coloop of $M$, $t(M, x, y)=t(M-e, x, y)+t(M \cdot e, x, y)$, where $M-e$ (respectively: $M \cdot e$ ) denotes the matroid obtained from $M$ by the deletion (respectively: contraction) of $e$.

(iii) If $M$ is the direct sum of the matroids $M_{1}$ and $M_{2}, t(M, x, y)=$ $t\left(M_{1}, x, y\right) t\left(M_{2}, x, y\right)$.

Another property of interest is that if $M$ and $M^{*}$ are dual matroids, $t\left(M^{*}, x, y\right)=t(M, y, x)$.

The following formula is due to Greene [4]. We shall provide a short proof for the sake of completeness.

Proposition 7. Let $\mathscr{C}$ be a subspace of $\mathrm{GF}(q)^{|E|}$ and $r$ be the rank-function of $M(\mathscr{C})$.

(i) $\sum_{K \in \epsilon_{\perp}} z^{s(K)}=z^{|E|-r(E)}(1-z)^{r(E)} t(M(\mathscr{C}),(1+(q-1) z) /(1-z), 1 / z)$. $\left(\mathrm{i}^{*}\right) \quad \sum_{C \in f^{f}} z^{s(C)}=z^{r(E)}(1-z)^{|E|-r(E)} t(M(\mathscr{C}), 1 / z,(1+(q-1) z) /(1-z))$.

Proof. Consider the polynomial $P(v)=\sum_{K \in \mathcal{F}_{\perp}}(1+v)^{|E|-s(K)}$ in the variable $v$. Clearly

$$
P(v)=\sum_{K \in \mathcal{K}^{\prime} \perp} \sum_{F \subseteq E-S(K)} v^{|F|}=\sum_{F \subseteq E} \sum_{K \in \mathcal{F} \perp, S(K) \subseteq E-F} v^{|F|} .
$$

Let $I$ be a spanning independent subset of $F$ and $B$ be a basis of $M(\mathscr{C})$ which contains $I$. For $K \in \mathscr{C}^{\perp}, S(K) \subseteq E-F$ iff the restriction of $K$ to $I$ is zero. Considering the fundamental basis of the space $\mathscr{C}^{\perp}$ associated to $B$ it is clear that the number of elements $K \in \mathscr{C}^{\perp}$ such that $S(K) \subseteq E-F$ is 


$$
\begin{aligned}
q^{|B|-|I|} & =q^{r(E)-r(F)} . \text { Hence } P(v)=\sum_{F \subseteq E} q^{r(E)-r(F)} v^{|F|} \\
& =v^{r(E)} \sum_{F \subseteq E}(q / v)^{r(E)-r(F)} v^{|F|-r(F)}=v^{r(E)} t(M(\mathscr{C}), 1+(q / v), 1+v) .
\end{aligned}
$$

Taking $1+v=1 / z$ yields formula $(\mathrm{i})$. Formula $\left(\mathrm{i}^{*}\right)$ follows by duality.

Now, coming back to ternary matroids, let $\mathscr{C}$ be a subspace of $\operatorname{GF}(3)^{|E|}$ and $r$ be the rank-function of $M(\mathscr{C})$. Taking $q=3$ and $z=j$ in formula $\left(i^{*}\right)$ of Proposition 7 yields $f(\mathscr{C})=\sum_{C \in \mathscr{E}} j^{s(C)}=j^{r(E)}(1-j)^{|E|-r(E)} t\left(M(\mathscr{C}), j^{2}, j\right)$. Comparing with Proposition 6 and noting that $|E|-r(E)=\operatorname{dim} \mathscr{C}$, we obtain:

$$
j^{|E|-\operatorname{dim} ₹}(1-j)^{\operatorname{dim} \hbar} t\left(M(\mathscr{C}), j^{2}, j\right)=\varepsilon(\mathscr{C})\left(j-j^{2}\right)^{\operatorname{dim} \mathscr{C}+d(\mathscr{C})} .
$$

This formula and its complex conjugate yield:

Proposition 8. For any ternary space $\mathscr{C}$ on $E$ :

(i) $t\left(M(\mathscr{C}), j^{2}, j\right)=\varepsilon(\mathscr{C})\left(j^{2}\right)^{\operatorname{dim} \mathscr{F}+|E|}\left(j-j^{2}\right)^{d(\mathscr{F})}$;

(ii) $t\left(M(\mathscr{C}), j, j^{2}\right)=\varepsilon(\mathscr{C}) j^{\operatorname{dim} \mathscr{f}+|E|}\left(j^{2}-j\right)^{d(\mathscr{C})}$ with $\varepsilon(\mathscr{C}) \in\{1,-1\}$. The modulus of $t\left(M(\mathscr{C}), j, j^{2}\right)$ is $(\sqrt{ } 3)^{d(\mathscr{E})}$.

\section{CONCLUDING REMARKS}

The unicity of ternary representations [2] allows us to associate to every ternary matroid $M$ the dimension $d(M)$ of the bicycle space for any representation of $M$ over GF(3). By Proposition $8, d(M)$ depends only on the Tutte polynomial of $M$.

In fact we may define a signature mapping $\varepsilon$ from the class of all ternary matroids to $\{1,-1\}$ by setting $\varepsilon(M(\mathscr{C}))=\varepsilon(\mathscr{C})$ for every ternary space $\mathscr{C}$ (the consistency of this definition follows from Proposition 8). We can also define the quadripartition of $M$ as follows: the type of the element $e$ is $(2 a)$ if it is a loop, $(2 b)$ if it is a coloop, and otherwise depends on the values of the invariant $d$ on $M, M-e$ and $M \cdot e$ as specified in Proposition 2.

Proposition 9. For every ternary matroid $M$ on $E$ with dual $M^{*}$ :

(i) $\varepsilon(M)=(-1)^{d(M)} \varepsilon\left(M^{*}\right)$;

(ii) if $|E| \geq 2, \varepsilon(M)=\varepsilon(M \cdot e)$ if $e$ is a loop or is of type (1) or (2b) with respect to $M$, and $\varepsilon(M)=-\varepsilon(M \cdot e)$ otherwise;

(iii) if $|E| \geq 2, \varepsilon(M)=\varepsilon(M-e)$ if and only if $e$ is of type $(2 a)$ or $(2 b)$ with respect to $M$.

Proof. Noting that $t\left(M, j, j^{2}\right)$ and $t\left(M^{*}, j, j^{2}\right)$ are complex conjugates, we easily deduce (i) from Proposition 8. Statement (ii) is just a reformulation of Proposition 5. Finally (iii) is easily obtained from (ii) by duality, using (i) and Proposition 2.

It is not difficult to see that Proposition 8 follows by induction (using the recursive definition of the Tutte polynomial given in Section 3 ) from Proposition 
9. Our proof of the existence of a signature $\varepsilon$ satisfying Proposition 9 uses the weight enumerator $f$. It would be interesting to have a more direct proof, either by showing the consistency of (i), (ii), (iii) considered as axioms, or by finding a simpler interpretation of $\varepsilon$.

Finally we note that a simple necessary condition for a matroid $M$ to be ternary is that the modulus of $t\left(M, j, j^{2}\right)$ be of the form $(\sqrt{3})^{d}$ for some integer $d$ with $0 \leq d \leq \min \left\{r(M), r\left(M^{*}\right)\right\}$, where $r$ denotes rank.

\section{REFERENCES}

1. T. H. Brylawski, A decomposition for combinatorial geometries, Trans. Amer. Math. Soc. 171 (1972), 235-282.

2. T. H. Brylawski and D. Lucas, Uniquely representable combinatorial geometries, Proc. of Int. Colloq. in Combinatorial Theory, Rome, Italy, 1973; Atti Dei Convegni Lincei 17, Tomo 1 (1976), 83-104.

3. H. Crapo, The Tutte polynomial, Aequationes Math. 3 (1969), 211-229.

4. C. Greene, Weight enumeration and the geometry of linear codes, Studies in Applied Math. 55 (1976), 119-128.

5. L. H. Kauffman, New invariants in the theory of knots, Amer. Math. Monthly 95 (1988), no. 3, 195-242.

6. W. B. R. Lickorish and K. C. Millett, Some evaluations of link polynomials, Comment. Math. Helvetici 61 (1986), 349-359.

7. M. J. MacWilliams and N. J. A. Sloane, The theory of error-correcting codes, North-Holland, Amsterdam, New York, Oxford, 1978.

8. P. Rosenstiehl and R. C. Read, On the principal edge tripartition of a graph, Annals of Discrete Math. 3, 195-226.

9. M. B. Thistlethwaite, A spanning tree expansion of the Jones polynomial, Topology 26 (1987), 297-309.

10. W. T. Tutte, A ring in graph theory, Proc. Cambridge Philos. Soc. 43 (1947), 26-40.

11. _ A contribution to the theory of chromatic polynomials, Canad. J. Math. 6 (1954), 80-91.

12. D. J. A. Welsh, Matroid theory, Academic Press, London, 1976.

Laboratoire de Structures Discrètes, IMAG, BP 53 X, 38041 Grenoble Cédex, France 